\title{
Transmission of cytomegalovirus in fresh and freeze-thawed mother's own milk to very preterm infants: a cohort study
}

\author{
Christina Volder ${ }^{1,2,5}$, Benedicte Juul Work (D) ${ }^{1,2,5}$, Silje Vermedal Hoegh ${ }^{3}$, Maria-Christina Eckhardt ${ }^{4}$ and Gitte Zachariassen (iD ${ }^{1,2,4}{ }^{凶}$
}

(c) The Author(s), under exclusive licence to Springer Nature America, Inc. 2021

OBJECTIVES: The objectives of the study were to clarify: (i) the frequency of human cytomegalovirus (HCMV) transmission, (ii) the association between the viral load in mother's own milk (MOM), the amount of fresh MOM and transmission, and (iii) the frequency of sepsis-like-symptoms (SLS) among infants born to seropositive mothers compared to infants born to seronegative mothers. STUDY DESIGN: This prospective cohort study enrolled very preterm infants (gestational age <32 weeks) from Denmark. Weekly samples of fresh MOM and urine were analyzed for HCMV-DNA.

RESULTS: Twenty-six very preterm infants were enrolled. Four acquired an HCMV infection, of which two developed SLS. HCMVinfected infants received MOM with a significant higher viral load compared to the HCMV-uninfected infants.

CONCLUSION: A combination of a high viral load and an increased amount of fresh MOM increased the risk of HCMV transmission. SLS was only slightly more common among infants exposed to HCMV positive MOM.

Journal of Perinatology (2021) 41:1873-1878; https://doi.org/10.1038/s41372-021-01129-z

\section{INTRODUCTION}

Mother's own milk (MOM) is considered the optimal nutrition for very preterm infants [gestational age (GA) $<32$ weeks]. Unfortunately, MOM can contain viruses, which can be transmitted to very preterm infants through enteral feeding. The most common postnatal viral infection is caused by the human cytomegalovirus (HCMV). The HCMV infection can be either asymptomatic or can lead to serious clinical symptoms including cholestasis, pneumonitis, sepsis-like-symptoms (SLS), and even death [1-8]. Furthermore, an association between acquired HCMV-infection and long-term outcomes such as lower cognitive and motor function scores in infants has been described [9]. This study focuses on HCMV complicated by SLS.

Due to a local HCMV reactivation in the mammary gland postpartum HCMV seropositive mothers can secrete the virus in MOM and transmit it to the infants [5]. The extent of the HCMV transmission is dependent on whether MOM is fresh, freeze-thawed, pasteurized, or treated otherwise. Freezing of MOM is shown to reduce the viral load and thereby reduce the risk of transmission, where long-term pasteurization completely eliminates the virus [10-12].

SLS are a common term to describe a severe HCMV infection $[1,13-15]$. However, neonatal sepsis is common among all preterm infants [16]. Only few studies have compared the frequency of SLS in infants with a postnatal HCMV infection with a control group, and they found no significant difference [1, 17]. The fear of SLS due to postnatal HCMV infection has led to national recommendations in several countries recommending to process MOM (e.g., pasteurization or freezing) [13]. In Austria, freeze-thawing is recommended, Germany do not recommend pasteurization, Switzerland has no official guidelines and we have not been able to find official American guidelines. Likewise, no official guidelines are present in Denmark, where a combination of fresh and freeze-thawed milk is used to feed very preterm infants [18]. It is not clarified, how this combination of MOM affects the transmission from the mother to the infant.

The aim of this study was to provide further information about postnatal HCMV infection among very preterm infants in a level III NICU (neonatal intensive care unit) in Denmark, in order to make suggestions for future processing of MOM. Specifically, in infants born to HCMV seropositive mothers we examined: (i) the frequency of the transmission of HCMV from mother to infant, (ii) the association between the viral load in MOM, the amount of fresh milk and the transmission of HCMV to the infant, and (iii) the frequency of SLS among infants exposed to virus in MOM compared to a control group consisting of infants born to HCMV seronegative mothers.

\section{METHOD \\ Study design and population}

This prospective cohort study enrolled a population consisting of mothers and their preterm infants admitted to the NICU at Odense University Hospital, Denmark from April 2019 to January 2020. The study included very preterm infants, born with a GA $<32$ weeks and their mothers from the catchment area of Funen. Eligible patients were approached by members of the research team 1 to 7 days postpartum. Exclusion criteria were congenital HCMV infection and if the preterm infant was not fed fresh or freeze-thawed MOM, but only donor milk (pasteurized milk from other women) or preterm formula. The participants were followed for a minimum of 4 weeks.

\footnotetext{
${ }^{1}$ Institute of Clinical Research, University of Southern Denmark, Odense, Denmark. ${ }^{2}$ OPEN-Open Patient data Explorative Network, Odense University Hospital, Region of Southern Denmark, Odense, Denmark. ${ }^{3}$ Department of Clinical Microbiology, Odense University Hospital, Odense, Denmark. ${ }^{4} \mathrm{H} . \mathrm{C}$. Andersen Children's Hospital, Odense University Hospital, Odense, Denmark. ${ }^{5}$ These authors contributed equally: Christina Volder, Benedicte Juul Work. ${ }^{凶}$ email: gitte.zachariassen@rsyd.dk
} 


\section{Data collection}

HCMV screening. Within 7 days postpartum, a saliva sample from the infant and a maternal blood sample were collected. The saliva sample was used to screen the infant for congenital HCMV infection. In case of a positive saliva sample, a urine sample was collected to confirm congenital HCMV infection [19]. The maternal blood sample was screened for HCMV IgG by serology. If the maternal blood sample was IgG positive, samples of urine from the infant and fresh MOM were collected once a week starting at day 7-10 postpartum. In case of insufficient urine production, saliva samples were taken, until urine production became sufficient. If the maternal blood sample was IgG negative, no urine and milk samples were taken. Infants born to seronegative women comprised the control group.

Polymerase chain reaction (PCR). The milk, urine, and saliva samples were screened for HCMV by PCR. DNA was extracted from $500 \mu \mathrm{l}$ sample material and analyzed by an inhouse TaqMan real-time PCR. The real-time PCR assay UL55 used for HCMV detection and quantification has been described previously [20]. Quantification of positive samples was done by the inclusion of a standard curve and stated in copies $/ \mathrm{ml}$. Standard curves were constructed from extracted DNA from patient samples. Limit of quantification was 1445 copies/ml for HCMV. However, limits of detection of both assays were somewhat lower. Results below 1000 copies/ml were reported as 1000 copies $/ \mathrm{ml}$. The analyses were performed by the Department of Microbiology at Odense University Hospital.

Neonatal outcome variables. During admission, when the neonatologist who were treating the infants regardless of the study suspected sepsis, the infant's symptoms were registered in the medical file, and markers of infection [C-reactive protein (CRP) and leukocytes] were taken. Whenever markers of infection were taken, the researchers screened the medical files for SLS leading to the suspicion of sepsis. SLS was defined as three or more of the following clinical symptoms, in the absence of a positive blood culture: Brady- or tachycardia, episodes with apnea or increased need of oxygen [in ventilator (including noninvasive), in nasal-CPAP, or high-nasal flow], hypotonia or seizures, irritability or lethargy, and poor skin color or capillary response above $3 \mathrm{~s}$. Furthermore, paraclinical values were registered. It was noted whether SLS correlated with a positive HCMV urine sample. The medical files were screened by two researchers independently.

\section{Local procedures and guidelines}

In our NICU, enteral feeding of the preterm infant starts within the first day of life. Human colostrum and transitional milk (MOM from the first 14 days postpartum) is given to the infant as fresh milk. Any leftovers are frozen for later use, as this milk contains a higher level of protein compared to mature milk (MOM from 2 weeks postpartum) [21]. After 2 weeks the freeze-thawed MOM ( $~ 80 \%$ of the daily amount) will be given to the infant in combination with fresh MOM ( $20 \%$ of the daily amount), until freeze-thawed MOM from the first 14 days is no longer available. Afterwards, primarily fresh MOM is used. During hospitalization, donor milk or preterm formula will be given to the infant, if MOM is insufficient. All donor milk has been pasteurized and blood products for blood transfusions are leukodepleted and irradiated, which both prevent a postnatal HCMV transmission through donor milk and blood products. The distribution of parenteral nutrition, donor milk, preterm formula, fresh MOM, and freeze-thawed MOM is registered by the nurses in the infants' fluid chart. Data on the distribution was collected from these charts.

According to local guidelines, infants with an acquired HCMV infection demonstrating symptomatic disease will be treated symptomatically or with antiviral therapy in case of signs of organ failure due to HCMV.

\section{Data storage}

The data were stored securely using the Research Electronic Data Capture (REDCap) by the research infrastructure Odense Patient data Exploration Network (OPEN). Data were entered using an encrypted connection and was hereby fulfilling the demand for data security.

\section{Statistical analysis}

Statistical significance was tested using Fisher's exact test and two-sample Wilcoxon rank-sum test due to small numbers of observations, using Stata version 16 (StataCorp, College Station, TX). A $P$ value $<0.05$ was considered significant in all analyses. In the event of missing data or dropout before 4 weeks of sampling, the data were ignored and not a part of the analyses.

\section{RESULTS}

\section{Study population}

During the study period, a total of 26 preterm infants and their 22 mothers were enrolled and fulfilled the inclusion criteria with at least 4 weeks of sampling (Fig. 1). Mean GA was $28+4$ weeks and days (95\% confidence interval (CI) $27+4-29+4$ weeks and days). Mean birth weight (BW) was $1155 \mathrm{~g}$ (95\% Cl 986-1325 g). No infants had a positive saliva sample indicating congenital HCMV infection. Of the 22 mothers screened for HCMV by serology, 13 were IgG positive (59\%), indicating previous HCMV infection, and therefore the possibility of viral reactivation in the mammary gland. The mean observation time was 69 days $(95 \% \mathrm{Cl}$ 60-79 days).

\section{HCVM in MOM and urine samples}

As shown in Fig. 1, 12 out of 13 HCMV seropositive mothers had detectable HCMV in MOM. The individual viral loads (copies $/ \mathrm{ml}$ ) in MOM are shown in Fig. 2. The mean time for the first detectable HCMV in MOM was 15 (7-35) days. A total number of 271 milkand urine samples were collected with only 21 sample results missing.

\section{Transmission of HCMV from seropositive mothers to the infants}

Of the 16 infants born to seropositive mothers, four had a positive HCMV urine sample (HCMV-infected infants), and 12 had only negative HCMV urine samples (HCMV-uninfected infants) during the study period. Therefore, the frequency of postnatal HCMV transmission was $25 \%$ (Fig. 1). The mean individual maximum viral load in fresh MOM was 65,675 copies $/ \mathrm{ml}$ (95\% Cl 40,639-90,711 copies/ml) given to the four HCMV-infected infants and 25,366 copies/ml $(95 \%$ $\mathrm{Cl}-5102-55,834$ copies $/ \mathrm{ml}$ ) given to the 12 HCMV-uninfected infants. The difference between the two distributions was significant ( $p$ value 0.02 ). The correlation between the viral load in fresh MOM and the amount of fresh MOM given to infected and uninfected HCMV infants respectively is illustrated in Fig. 3. There was no significant difference in GA or BW comparing the HCMV-infected and HCMV-uninfected infants (Table 1).

\section{Sepsis-like-symptoms}

A total of 12 infants demonstrated SLS during hospitalization. Episodes of SLS among infants born to seronegative and seropositive mothers, respectively, are shown in Table 2. The number of infants with SLS and the average number of SLS

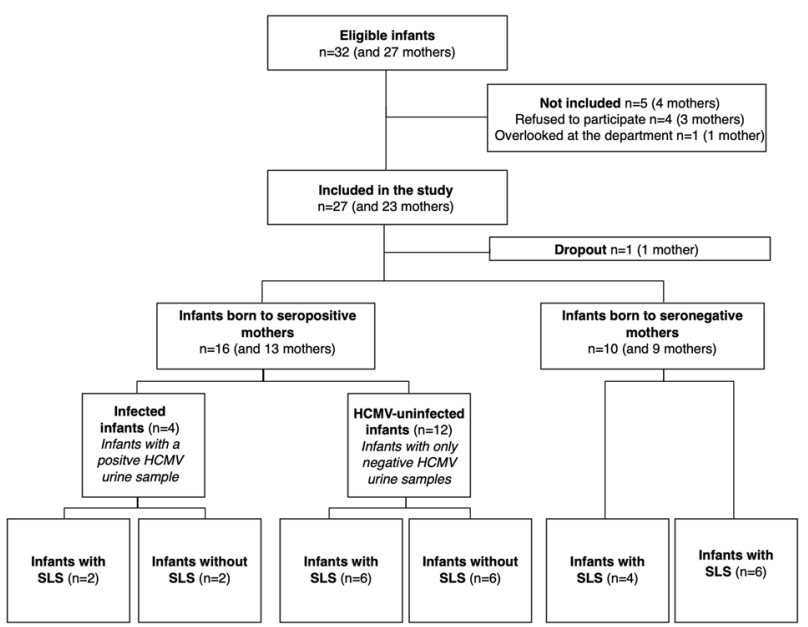

Fig. 1 Flowchart of the study. Flowchart describing the enrollment of infants and mothers, mother's seroprevalence, the secretion of virus in mother's own milk (MOM), and the transmission to the preterm infant. 


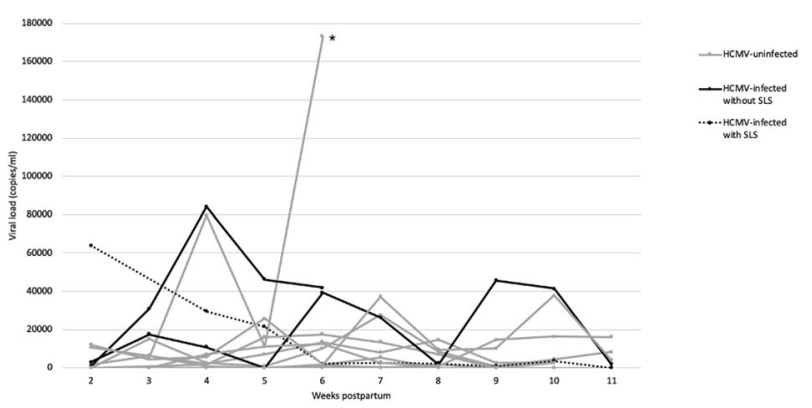

Fig. 2 Kinetics of human cytomegalovirus in fresh mother's own milk (MOM). The individual viral load of HCMV-DNA in fresh MOM shown with postpartum weeks until 11 weeks postpartum. Asterisk $(*)$ indicates the infant received $<20 \%$ fresh MOM and died 5 weeks old due to short bowel syndrome.
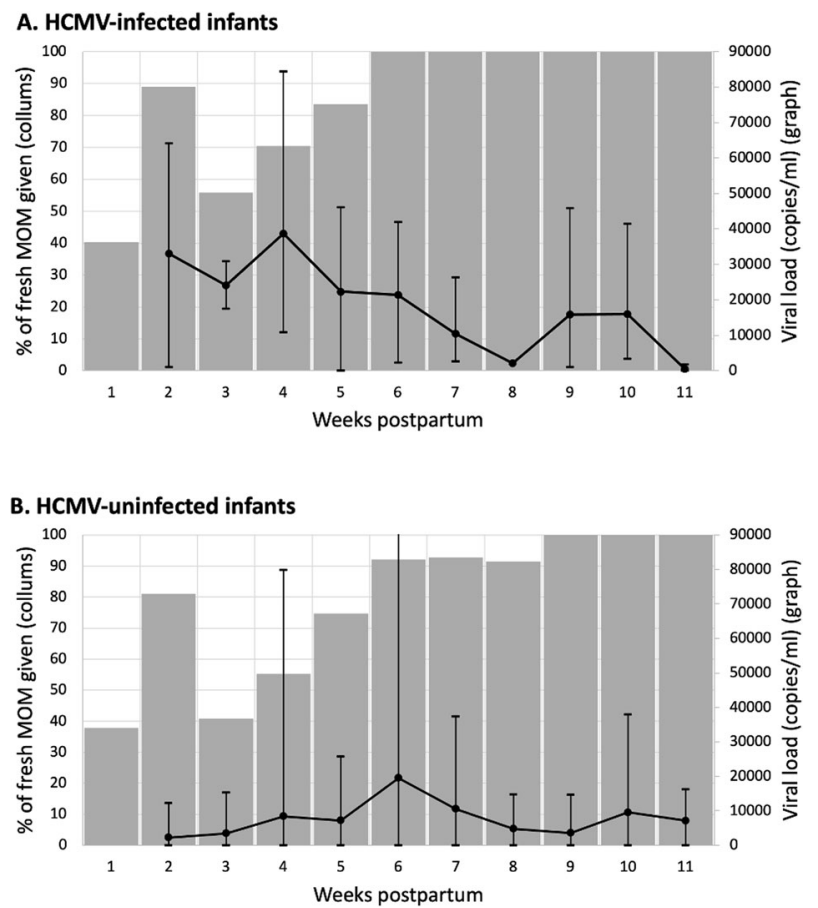

Fig. 3 HCMV in MOM and amount of fresh MOM. Correlation between viral load of HCMV in fresh MOM and the amount of fresh MOM given to (A) HCMV-infected and (B) HCMV-uninfected infants. The bar charts illustrate the mean percentage of fresh MOM. The line graphs illustrate the mean viral load in fresh MOM, including maximum and minimum.

episodes were only slightly higher among infants born to seropositive mothers compared to infants born to seronegative mothers. Due to the small number of HCMV-infected infants no statistical analysis was made to compare these infants with infants born to seronegative mothers. The two HCMV-infected infants who developed SLS were born extremely preterm with a GA of 25 +2 weeks and days; their courses are described in the following.

The two infants were twins; a boy (infant 10) and a girl (infant 11) with a BW of 880 and $770 \mathrm{~g}$, respectively. At 25 days of age infant 10 developed SLS with bradycardia, episodes with apnea, and lethargy. A urine sample from the day before was HCMV positive. All the following urine samples were HCMV positive. At 26 days of age infant 11 developed SLS, with bradycardia, episodes with apnea, lethargy, and poor skin color. A HCMV urine sample collected 5 days later was positive. Surprisingly, the three following urine samples were negative, followed by more positive samples. The negative samples could be explained by

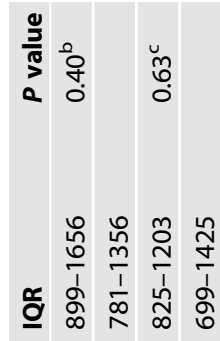


Table 2. Outcome variables registered in the medical files of 26 premature infants when markers of infection were taken.

\begin{tabular}{|c|c|c|c|c|c|c|c|}
\hline & \multicolumn{6}{|c|}{ Infants born to } & \multirow[t]{3}{*}{$P$ value } \\
\hline & \multicolumn{2}{|c|}{ Seronegative mothers } & \multicolumn{4}{|c|}{ Seropositive mothers } & \\
\hline & $\begin{array}{l}\text { All infants, } \\
n=10\end{array}$ & $95 \% \mathrm{Cl}^{\mathrm{a}}$ & $\begin{array}{l}\text { All infants, } \\
n=16\end{array}$ & $95 \% \mathrm{Cl}$ & $\begin{array}{l}\text { HCMV- } \\
\text { infected } \\
\text { infants, } n=4\end{array}$ & $\begin{array}{l}\text { HCMV-uninfected } \\
\text { infants, } n=12\end{array}$ & \\
\hline Infants with SLS,$n(\%)$ & $4(40)$ & & $8(50)$ & & $2(50)$ & $6(50)$ & $0.70^{c}$ \\
\hline Episodes of SLS, $n$ (average) & $5(0.50)$ & $-0.06-10.06$ & $13(0.81)$ & $4.08-21.92$ & $4(1.00)$ & $9(0.75)$ & $0.52^{d}$ \\
\hline \multicolumn{8}{|l|}{ Paraclinical abnormalities ${ }^{e}$} \\
\hline High CRP, $n(\%)$ & $1(20)$ & & $4(31)$ & & $1(25)$ & $3(33)$ & \\
\hline Slightly elevated CRP, $n$ (\%) & $1(20)$ & & $6(46)$ & & $2(50)$ & $4(44)$ & \\
\hline Thrombocytosis, $n$ (\%) & $3(60)$ & & $6(46)$ & & $3(75)$ & $3(33)$ & \\
\hline Thrombocytopenia, $n$ (\%) & 0 & & $1(8)$ & & 0 & $1(11)$ & \\
\hline \multicolumn{8}{|c|}{$\begin{array}{l}\text { a95\% confidence interval. } \\
\text { bSepsis-like-symptoms. } \\
\text { cFisher's exact test comparing infants born to HCMV seropositive mothers and infants born to HCMV seronegative mothers. } \\
\text { d Two-sample Wilcoxon rank-sum comparing infants born to HCMV seropositive mothers and infants born to HCMV seronegative mothers. } \\
\text { eParaclinical abnormalities registered when an episode of SLS was present: high c-reactive protein }(>49 \mathrm{mg} / \mathrm{l}) \text {, slightly elevated c-reactive protein }(6-49 \mathrm{mg} / \mathrm{l}) \text {, } \\
\left.\text { thrombocytosis }\left(>350 \times 10^{9} / \mathrm{l}\right) \text {, thrombocytopenia }\left(20 \times 10^{9} / \mathrm{l}\right) \text {, leukopenia }(40 \mathrm{U} / \mathrm{l}) \text {, gamma-glutamyl-transferase }(>150 \mathrm{U} / \mathrm{l}) \text {, or basic phosphatase }(>700 \mathrm{U} / \mathrm{l})\right) \text {. }\end{array}$} \\
\hline
\end{tabular}

urine samples with a viral load around the limit of detection. Infant 11 had one more episode at the age of 40 days with increased need of oxygen, hypotonia, lethargy and poor skin color, and one more episode at the age of 60 days with tachypnea, hypotonia, lethargy, and poor skin color. Both twins responded well to treatment with paracetamol and caffeine citrate. The infants were not treated with antibiotics, and there was no indication for antiviral treatment. During the first 6 weeks of life the twins were fed $75 \%$ fresh MOM which contained a high viral load up to 64,100 copies $/ \mathrm{ml}$.

\section{DISCUSSION}

This cohort study assessed the maternal seroprevalence and the risk of postnatal HCMV transmission in 26 very preterm infants. We found that the transmission of HCMV depended on the viral load in fresh MOM and the amount of fresh MOM. Furthermore, SLS was slightly more common among infants exposed to HCMV positive MOM, but the difference was not significant.

A total of $59 \%$ of the mothers in this study were HCMV seropositive. This is consistent with previous literature, which reports a seroprevalence that range from 40 to $60 \%$ in the United States and Western European Countries [6, 13].

Previous studies report a high reactivation rate (up to $100 \%$ ) of HCMV during lactation using PCR detection [4, 6, 10, 12]. In agreement with this, we found that 12 out of 13 seropositive mothers had detectable HCMV in MOM. Only one mother never had detectable HCMV in MOM, which could be explained by milk samples with a viral load around the limit of detection.

The secretion of HCMV in MOM is reported to differ between individuals, which our results support. However, not all samples follow the unimodal kinetic, which is reported as the most common [6].

The use of freeze-thawed MOM in our NICU probably has an impact on the viral load of HCMV transmitted to the infant. An Iranian study reports that freezing at $-20^{\circ} \mathrm{C}$ for $72 \mathrm{~h}$ reduces the viral load significantly, and completely eliminates HCMV in $84 \%$ of the milk samples [11]. In addition, a Spanish study reports that freezing reduces the rate of HCMV transmission by $78 \%$, and number needed to treat to prevent a transmission is 23 [10].

An association between an increased transmission of HCMV and a high viral load in MOM are reported in previous studies $[1,22]$. In accordance with this, we found that the mean individual maximum viral load in fresh MOM was significantly higher among the HCMV-infected infants compared to the HCMV-uninfected infants. However, some infants received fresh MOM with a high viral load but remained HCMV-uninfected. This could be explained by the fact that these infants received mostly freeze-thawed MOM or parenteral nutrition, and therefore actually received a smaller amount of virus, which prevented transmission. One infant received MOM with a maximum viral load at 173,000 copies $/ \mathrm{ml}$ and remained noninfected. The infant received $<20 \%$ fresh MOM, and there were only 5 weeks of sampling due to death (early focal intestinal perforation and short bowel syndrome). This could explain, why the infant never became infected.

The results of this study suggest that a small percentage of fresh MOM from seropositive mothers lowers the risk of HCMV transmission. In correlation with this, a meta-analysis of 17 observational studies found that the transmission was significantly higher in preterm infants fed only fresh MOM, compared to freeze-thawed MOM and combinational diet [23]. However, a Swedish randomized controlled study did not find a significant difference in the risk of transmission among infants fed either fresh or freeze-thawed MOM, but the study lacked power [24].

Overall, the transmission rate in our study was $25 \%$. Previous studies report a transmission rate ranging from 5.7 to $58.6 \%$, depending on the processing of MOM [15]. Our results are in accordance with this, using a combination of fresh and freeze-thawed MOM in our NICU. We found no association between the transmission rate and GA or BW, respectively. Some studies are in accordance with this result [7, 17, 25], where others report that HCMV-infected infants are smaller and more immature $[1,10]$. 
The rate of SLS among preterm infants with a postnatal HCMV infection is often reported to a range from 0 to $25 \%$ [1, 13-15]. Our study found that two out of four of the HCMV-infected infants demonstrated SLS. The remaining two infants had a milder or an asymptomatic HCMV infection. The two infants demonstrating SLS were born extremely preterm. This, along with the small sample size of this study, may explain the high frequency of SLS among HCMV-infected infants, as preterm infants born at GA $<26$ weeks have an $80 \%$ risk of developing SLS, according to studies [13]. In general, preterm infants born at $\mathrm{GA}<28$ weeks are in greater risk of a severe symptomatic HCMV infection, due to the lack of placental transfer of protective antibodies, where the majority is transferred after the 28th week of pregnancy $[7,15]$.

There are a limited number of studies comparing the frequency of SLS among infants exposed to HCMV in MOM or HCMV-infected infants with a control group. In accordance with our findings, these studies find no significant difference in SLS [1, 17]. However, it must be taken into consideration that our sample size is very small, which increases the probability of type II errors. Previous studies found significant differences in paraclinical values $[1,7,26]$. In this study, paraclinical values during episodes of SLS were registered (Table 2), but due to few and vary outcomes these results are of minor use.

The strengths of the study were the presence of a control group consisting of infants born to seronegative mothers and detailed documentation of enteral and parenteral nutrition. The main limitation of this study was the small sample size. In addition, the clinicians who registered the infant's symptoms in the medical files were not blinded, but they were independent of the study. Moreover, weekly testing for HCMV in infant's urine and MOM in the infants born to seronegative mothers, would have added more strength to the study.

Several Western European countries have guidelines recommending processing of fresh MOM to preterm infants i.e., by freezing or pasteurization [13]. Even though freezing reduces the risk of transmission, studies argue that freezing of MOM is insufficient to eliminate transmission of HCMV and severe symptomatic HCMV infections [6, 27]. Pasteurization is a sufficient method to remove HCVM from MOM. But, on the other hand, it is a challenge to conserve the positive nutritional and immunological properties, which are important in order to e.g., support the development of the immature gut $[13,28]$. Maschmann et al. demonstrated that short-term heat treatment for $5 \mathrm{~s}$ at $62{ }^{\circ} \mathrm{C}$ may be the best compromise to reduce the viral load, with HCMV inactivation at $99.8-100 \%$, and at the same time preserve some enzyme activity and antibody binding [29]. It must be taken into consideration whether the benefits of feeding fresh MOM outweigh the risk of HCMV transmission and severe symptomatic HCMV infection. Freeze-thawing instead of pasteurizing MOM and feeding small amounts of fresh milk seemed in our study to reduce HCMV transmission. We found no significant difference in SLS and none of the infected infants developed organ failure due to HCMV. However, it cannot be ignored that HCMV can induce severe symptoms in preterm infants, especially those born at GA $<28$ weeks. Therefore, we recommend at least screening of all mothers giving birth at $\mathrm{GA}<28$ weeks, to increase the awareness of HCMV as a possible reason to SLS. There is not yet enough evidence to recommend either fresh or freeze-thawed MOM. However, feeding only or mainly freeze-thawed MOM could be considered in cases with a high viral load in MOM.

\section{CONCLUSION}

This study found the postnatal transmission rate of HCMV from mother to infant to be $25 \%$. Furthermore, the results of this study confirmed that the combination of a high viral load and an increased amount of fresh MOM increased the risk of HCMV transmission to the very preterm infant. There was no significant increase in SLS in infants exposed to HCMV in MOM compared to non-exposed infants.

Further and larger studies are needed to determine, whether infants exposed to HCMV via MOM, especially HCMV-infected infants, are in greater risk of SLS.

\section{REFERENCES}

1. Martins-Celini FP, Yamamoto AY, Passos DM, do Nascimento SD, Lima EV, Di Giovanni CM, et al. Incidence, risk factors, and morbidity of acquired postnatal cytomegalovirus infection among preterm infants fed maternal milk in a highly seropositive population. Clin Infect Dis. 2016;63:929-36.

2. Wakabayashi H, Mizuno K, Kohda C, Negoro T, Maekawa C, Sawato S, et al. Low HCMV DNA copies can establish infection and result in significant symptoms in extremely preterm infants: a prospective study. Am J Perinatol. 2012;29:377-82.

3. Meier J, Lienicke U, Tschirch E, Kruger DH, Wauer RR, Prosch S. Human cytomegalovirus reactivation during lactation and mother-to-child transmission in preterm infants. J Clin Microbiol. 2005;43:1318-24.

4. Lombardi G, Garofoli F, Manzoni P, Stronati M. Breast milk-acquired cytomegalovirus infection in very low birth weight infants. J Matern Fetal Neonatal Med. 2012;25:57-62.

5. Hamprecht K, Maschmann J, Vochem M, Dietz K, Speer CP, Jahn G. Epidemiology of transmission of cytomegalovirus from mother to preterm infant by breastfeeding. Lancet. 2001;357:513-8.

6. Hamprecht K, Goelz R. Postnatal cytomegalovirus infection through human milk in preterm infants: transmission, clinical presentation, and prevention. Clin Perinatol. 2017;44:121-30.

7. Capretti MG, Lanari M, Lazzarotto T, Gabrielli L, Pignatelli S, Corvaglia L, et al. Very low birth weight infants born to cytomegalovirus-seropositive mothers fed with their mother's milk: a prospective study. J Pediatr. 2009;154:842-8.

8. Vochem M, Hamprecht K, Jahn G, Speer CP. Transmission of cytomegalovirus to preterm infants through breast milk. Pediatr Infect Dis J. 1998;17:53-8.

9. Bevot A, Hamprecht K, Krageloh-Mann I, Brosch S, Goelz R, Vollmer B. Long-term outcome in preterm children with human cytomegalovirus infection transmitted via breast milk. Acta Paediatr. 2012;101:e167-72.

10. Balcells C, Botet F, Gayete S, Marcos MA, Dorronsoro I, de Alba C, et al. Vertically transmitted cytomegalovirus infection in newborn preterm infants. J Perinat Med. 2016;44:485-90.

11. Hosseini M, Esmaili HA, Abdoli Oskouei S, Gojazadeh M, MokariYamchi Z, Layegh $V$, et al. Evaluation of the freeze-thawing method in reducing viral load of cytomegalovirus in breast milk of mothers of preterm infants. Breastfeed Med. 2016;11:557-60.

12. Hayashi S, Kimura H, Oshiro M, Kato $Y$, Yasuda A, Suzuki C, et al. Transmission of cytomegalovirus via breast milk in extremely premature infants. J Perinatol. 2011;31:440-5.

13. Bardanzellu F, Fanos V, Reali A. Human breast milk-acquired cytomegalovirus infection: certainties, doubts and perspectives. Curr Pediatr Rev. 2019;15:30-41.

14. Hamprecht K, Maschmann J, Jahn G, Poets CF, Goelz R. Cytomegalovirus transmission to preterm infants during lactation. J Clin Virol. 2008;41:198-205.

15. Kurath S, Halwachs-Baumann G, Muller W, Resch B. Transmission of cytomegalovirus via breast milk to the prematurely born infant: a systematic review. Clin Microbiol Infect. 2010;16:1172-8.

16. Shah BA, Padbury JF. Neonatal sepsis: an old problem with new insights. Virulence. 2014;5:170-8.

17. Jim WT, Shu CH, Chiu NC, Kao HA, Hung HY, Chang JH, et al. Transmission of cytomegalovirus from mothers to preterm infants by breast milk. Pediatr Infect Dis J. 2004;23:848-51.

18. Klotz D, Jansen S, Gebauer C, Fuchs H. Handling of breast milk by neonatal units: large differences in current practices and beliefs. Front Pediatr. 2018;6:235.

19. Boppana SB, Ross SA, Shimamura M, Palmer AL, Ahmed A, Michaels MG, et al. Saliva polymerase-chain-reaction assay for cytomegalovirus screening in newborns. N Engl J Med. 2011;364:2111-8.

20. Boeckh M, Huang M, Ferrenberg J, Stevens-Ayers T, Stensland L, Nichols WG, et al. Optimization of quantitative detection of cytomegalovirus DNA in plasma by real-time PCR. J Clin Microbiol. 2004;42:1142-8.

21. Radmacher $P G$, Lewis $S L$, Adamkin $D H$. Individualizing fortification of human milk using real time human milk analysis. J Neonatal Perinat Med. 2013;6:319-23.

22. van der Strate BW, Harmsen MC, Schafer $P$, Swart $P J$, The $T H$, Jahn $G$, et al. Viral load in breast milk correlates with transmission of human cytomegalovirus to preterm neonates, but lactoferrin concentrations do not. Clin Diagn Lab Immunol. 2001;8:818-21.

23. Lanzieri TM, Dollard SC, Josephson CD, Schmid DS, Bialek SR. Breast milk-acquired cytomegalovirus infection and disease in VLBW and premature infants. Pediatrics. 2013;131:e1937-45. 
24. Omarsdottir S, Casper C, Naver L, Legnevall L, Gustafsson F, Grillner L, et al. Cytomegalovirus infection and neonatal outcome in extremely preterm infants after freezing of maternal milk. Pediatr Infect Dis J. 2015;34:482-9.

25. Mussi-Pinhata MM, Yamamoto AY, do Carmo Rego MA, Pinto PC, da Motta MS, Calixto C. Perinatal or early-postnatal cytomegalovirus infection in preterm infants under 34 weeks gestation born to CMV-seropositive mothers within a high-seroprevalence population. J Pediatr. 2004;145:685-8.

26. Neuberger $P$, Hamprecht $K$, Vochem M, Maschmann J, Speer CP, Jahn G, et al. Case-control study of symptoms and neonatal outcome of human milktransmitted cytomegalovirus infection in premature infants. J Pediatr. 2006;148:326-31.

27. Maschmann J, Hamprecht K, Weissbrich B, Dietz K, Jahn G, Speer CP. Freezethawing of breast milk does not prevent cytomegalovirus transmission to a preterm infant. Arch Dis Child Fetal Neonatal Ed. 2006;91:F288-90.

28. O'Connor DL, Gibbins S, Kiss A, Bando N, Brennan-Donnan J, Ng E, et al. Effect of supplemental donor human milk compared with preterm formula on neurodevelopment of very low-birth-weight infants at 18 months: a randomized clinical trial. JAMA. 2016;316:1897-905.

29. Maschmann J, Muller D, Lazar K, Goelz R, Hamprecht K. New short-term heat inactivation method of cytomegalovirus (CMV) in breast milk: impact on CMV inactivation, CMV antibodies and enzyme activities. Arch Dis Child Fetal Neonatal Ed. 2019;0:604-8.

\section{ACKNOWLEDGEMENTS}

The authors thank the parents and infants for participating in the study. We also thank the nurses at the neonatal intensive care unit, Odense University Hospital, and the biomedical laboratory scientists and Rune Micha Pedersen and Marianne Skov at the Department of Clinical Microbiology, Odense University Hospital.

\section{AUTHOR CONTRIBUTIONS}

CV and BJW designed the study, collected data, carried out the analyses, drafted the paper, and revised the paper. They contributed equally as co-first authors. MCE collected data and reviewed the paper. SVH was responsible for the analyses methods and reviewed the paper. GZ conceptualized and designed the study, coordinated and supervised data collection, and critically reviewed the paper for important intellectual content. All authors approved the final paper as submitted and agree to be accountable for all aspects of the work.

\section{COMPETING INTERESTS}

The authors declare no competing interests.

\section{ETHICAL APPROVAL}

Written consents from the parents were obtained, after oral, and written information. The study was conducted in accordance with the declaration of Helsinki and approved by the local ethical committee in the region of Southern Denmark (S-20180187) and the data protection agency at Odense University Hospital (April 3rd, 2019).

\section{ADDITIONAL INFORMATION}

Correspondence and requests for materials should be addressed to G.Z.

Reprints and permission information is available at http://www.nature.com/ reprints

Publisher's note Springer Nature remains neutral with regard to jurisdictional claims in published maps and institutional affiliations. 